\title{
Effects of Errors on the Dynamic Aperture of the Advanced Photon Source Storage Ring*
}

\author{
H. Bizek, E. Crosbie, E. Lessner, L. Teng, J. Wirsbinski \\ Argonne National Laboratory \\ Advanced Photon Source \\ 9700 South Cass Avenue \\ Argonne, Il 60439
}

ANL $/$ CP -73090

DE91 011858

\section{Absiract}

The individual tolerance limits for alignment errors and magnet fabrication errors in the 7-GeV Advanced Photon Source storage ring are determined by computer-simulated tracking. Limits are established for dipole strength and roll errors, quadrupole strength and alignment errors, sextupole strength and alignment errors, as well as higher order multipole strengths in dipole and quadrupole magnets. The effects of girder misalignments on the dynamic aperture are also studied. Computer simulations are obtained with the iracking program RACETRACK, with errors introduced from a user-defined Gaussian distribution, truncated at \pm 5 standard deviation units. For each error, the average and rms spread of the stable amplitudes are determined for ten distinct machines, defined as ten different seeds to the random distribution, and for five distinct initial directions of the tracking particle.

\section{INTRODUCTION}

In this paper, the results of a systematic and detailed study of alignment and fabrication error effects on the dynamic aperture of the Advanced Photon Source (APS) storage ring are presented.

The APS is a 7-GeV synchrotron radiation source. Its storage ring has a Chasman-Green type lattice, with a circumference of $1104 \mathrm{~m}$, consisting of 40 cells, each containing one long zero-dispersion straight section. Figure 1 shows one section of the lattice, including the location of the beam position monitors and orbit-correcting magnets.

Magnet fabrication errors and alignment errors introduce distortions in the ideal orbit and cause a reduction of dynamic aperture, defined as the limiting stable betatron oscillation amplitude in either the horizontal or vertical plane of motion. Error fields in dipole magnets, roll angle misalignments and quadrupole displacements cause orbit distortions; quadrupole fields in dipole magnets, error field gradients in quadrupole magnets, and error quadrupole fields in sextupole magnets, cause detuning and changes in the

\footnotetext{
*Work supported by U.S. Department of Energy, Office of Basic Energy Sciences under Contract No. W-31-109-ENG-38.

${ }^{1}$ The damping time for a positron in the APS storage ring corresponds to 2,600 turns; 500 turns correspond to a damping factor of $\mathrm{e}^{(-500 / 2600)}=0.83$.
}

bctatron amplitude and dispersion functions. Dechromatizing effects can result from higher order error field gradients. Magnet misalignments included in this study were dipole and quadrupole rolls; quadrupole and sexiupole displacements; and magnet support girder displacements. Magnet fabrication errors included dipole, quadrupole. sextupole, and higher order multipole field errors in dipole and quadrupole magnets. The effects of these errors on orbit functions, before and after correction of the closed orbit, are reported in Reference [1].

Careful analysis of the results led to the establishment of individual tolerance limits of acceptable error levels in the storage ring.

\section{APERTURE REDUCTION}

The dynamic aperture limits in presence of errors were obtained numcrically using the tracking program RACETRACK [2] as modified by S. Kramer [3]. In the program, appropriate random errors can be introduced from a user-defined Gaussian distribution truncated at \pm 5 standard deviation units. A particle is considered stabie if it tracks for 500 turns. Damping effects are not included. ${ }^{1}$ For each case, 10 different "machines", corrasponding to 10 different seeds to the random number generator, were constructed, and their rms average values calculated. The process was repeated for several error levels and for 5 different initial coordinates of the tracking particle, parametrized by $\theta=$ $\tan ^{-1}(N Y / N X)$, where $N X$ and $N Y$ are the particle's oscillation amplitudes in the horizontal and vertical planes, respectively, expressed in units of the rms beam sizes $\sigma_{x}$ and $\sigma_{y}$, at the center of the long straight section, where they assume the values 0.342 and $0.203 \mathrm{~mm}$. The 5 initial directions were taken as those corresponding to $\theta$ equal to $2^{\circ}, 45^{\circ}, 90^{\circ}, 182^{\circ}$ and $225^{\circ}$.

Roll angle misalignments of dipole magnets cause vertical orbit distortions. A roll angle of $1.0 \mathrm{mrad} \mathrm{rms}$ reduced the dynamic aperture by a factor of 2 . horizontal or the vertical plane causes an error dipole field proportional to the product of the gradient field strength times the displacement. The large number and considerable strength of the quadrupole magnets together with the strong chromaticity correcting sextupoles make the APS storage ring very sensitive to quadrupole displacements. Indeed, of all the errors analyzed, these were the most restrictive: a horizontal displacement of $0.1 \mathrm{~mm} \mathrm{rms}$ reduces the dynamic aperture by $53 \%\left(\theta=45^{\circ}\right)$. Increasing the error level to

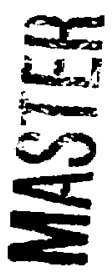




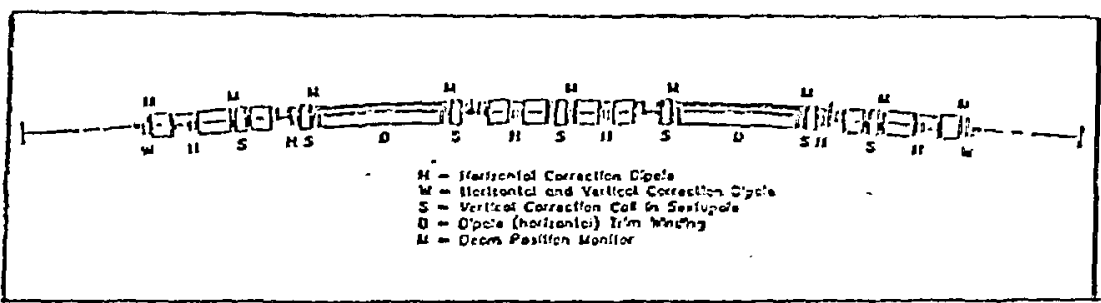

Figure 1. Layout of magnets for one cell of the APS storage ring.

$1.5 \mathrm{~mm}$ further reduces the dynamic aperture by $20 \%$, in addition to causing 2 unstable orbits out of the 10 processed. Horizontal and vertical displacements of $0.1 \mathrm{~mm} \mathrm{rms}$ each, considered together, reduce the dynamic aperture by $70 \%$ and cause relatively large dispersion at the center of the long straight section [1]. The effect of the horizontal quadrupole displacements on the dynamic aperture can be seen in Fig. 2, for 4 levels of errors, where we included the dynamic aperture for the ideal lattice, for comparison, and the rms spread corresponding to $0.1 \mathrm{~mm}$ displacements. NX and NY denote, as aforementioned, the horizontal and vertical stable amplitudes in units of the rms beam sizes.

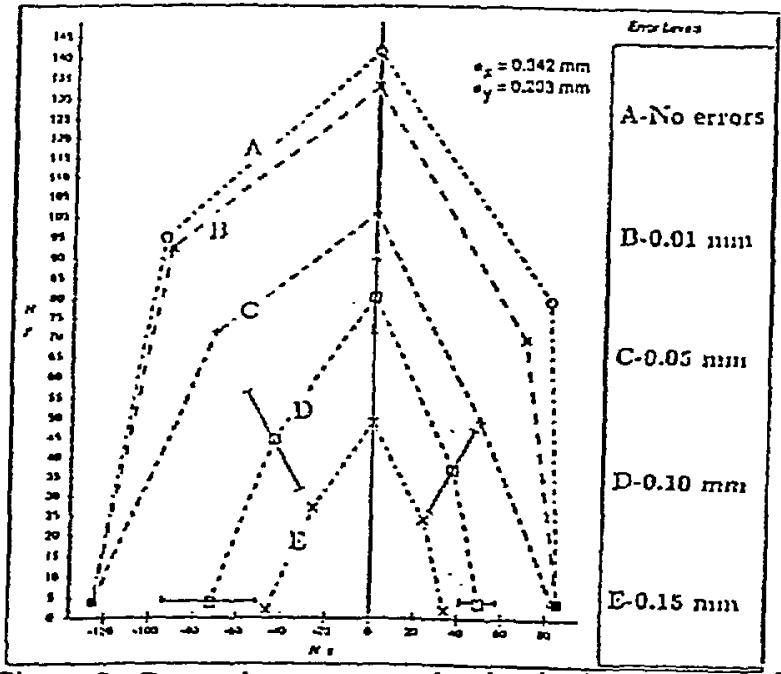

Figure 2. Dynamic aperture reduction in the presence of random horizontal quadrupole displacements, in units of the rms bean sizes $\sigma_{x}$ and $\sigma_{y}$ at the center of the straight section (see text).

Sextupole misalignments produced only small orbit distortions. Horizontal and vertical displacements of $0.2 \mathrm{~mm}$ rms, considered together, caused a $30 \%$ reduction of dynamic aperture.

The effects of error dipole fields in dipole magnets were found to be small, when compared to those of roll angle misalignments at the same error level (about one-third). For $\Delta \mathrm{B} / \mathrm{B}=1 \times 10^{-3}$, there was a $17 \%$ reduction of dynamic aperture along the $\theta=45^{\circ}$ direction.

Error field gradients in quadrupole magnets, $\Delta B^{\prime} / B^{\prime}$, induce detuning and distortions in the orbit functions. An

2The multipole coefficients are defined by: $B=B_{x}+i B_{y}=$ $B_{0} \Sigma_{n=0}^{\infty}\left(b_{n}+j a_{n}\right)(x+i y)^{n}$ off-set of $0.5 \%$ rms reduces the dynamic aperture by $56 \%$.

In Fig. 3 we display the reduction of the dynamic aperture for various levels of random dipole roll angle misalignments, quadrupole and sextupole magnet displacements, dipole strength errors and quadrupole strength errors. Also included in the figure are misalignments of the magnet support system, considered individually and with quadrupole displacements of $0.1 \mathrm{~mm}$ superimposed. As can be seen in the figure, dipole field errors of $1 \times 10^{-3}$ produce a much smaller reduction in the dynamic aperture than the reduction resulting irom dipole roll errors at the same level. The latice is not very sensitive to sextupo!e displacement enors. Also notice that magnet support misalignments are less restrictive than individual quadrupole misalignments by a factor of 5 .

Error sextupole fields in sextupole magnets are responsible for changes in chromatization, and have little effect on the dynamic aperture; at a high error level of $\triangle B^{\prime \prime} / B^{\prime \prime}=2 \times 10^{-2}$ rms the reduction was $30 \%$ along the $\theta=$ $45^{\circ}$ direction.

We also analyzed the effects in the dymamic aperture due to systematic and random higher-order multipole field errors in dipole and quadrupole magnets. Random quadrupole field errors in dipole magnets, with multipole coefficient ${ }^{2} b_{1}=$ $4 \times 10^{-4} / \mathrm{cm}$ produced a dynamic aperture reduction of $50 \%$. In quadrupole magnets, the same level of reduction occurred for random normal sextupole field errors with $b_{2}=$ $1 \times 10^{-3} / \mathrm{cm}^{2}$ and ranaiom octupole field errors with $b_{3}=$ $3 \times 10^{-4} / \mathrm{cm}^{3}$. The effects of systematic sextupole and octupole field errors in quadrupole magnets, with, respectively, $b_{2}=2 \times 10^{-3} / \mathrm{cm}^{2}$ and $b_{3}=1 \times 10^{-3} / \mathrm{cm}^{3}$, can be seen in Fig. 4.

We have so far investigated the effects on the dynamic aperture for individual errors. The effects of combined errors are being investigated. Based on the criterion of an allowed $50 \%$ reduction of dynamic aperture, we have established individual tolerance limits for magnet errors in the APS storage ring. These limits are shown in Table 1.

\section{CONCLUSIONS}

The presence of strong sextupole magnets in the latice cause the dynamic aperture to decrease sharply with orbit distorions.

The most restrictive cffects are those resulting from quadrupole misalignments, leading us to impose a somewhat tight tolerance of $0.1 \mathrm{~mm}$. A quadrupole misalignment of 


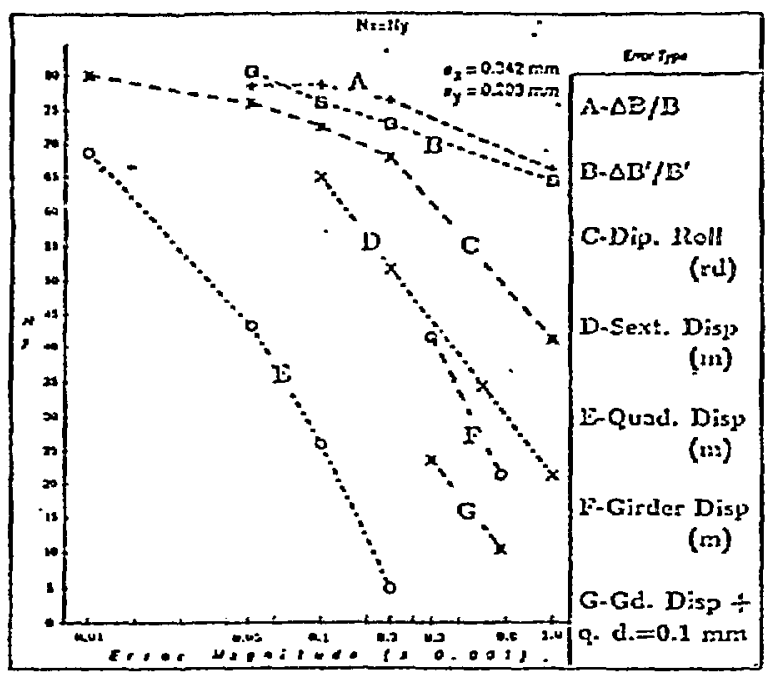

Figure 3. Dynamic aperture vs. error level for random dipole roll, quadrupole and sextupole magnet misalignments and displacements of the magnet support system, and dipole and quadrupole strength errors.

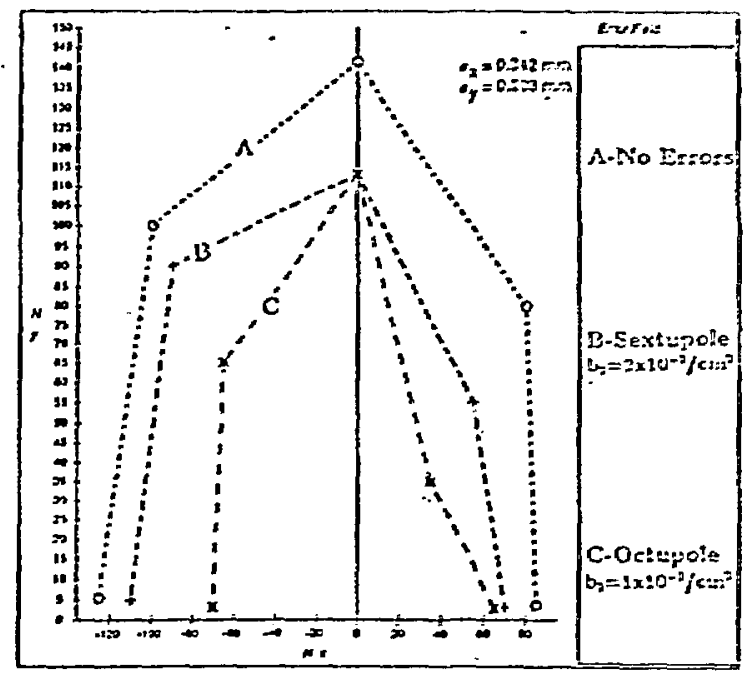

Figure 4. Effects of systematic sextupole and octupole field errors in quadrupole magnets, at the given multipole coefficients' levels.

Table 1

\section{Error Type}

Random roll angle misalignments of dipole magnets

Random horizontal or vertical displacements of quadrupole magnets

Random horizontal or vertical displacements of sextupole magnets

Random error dipole fields in dipole magnets

Random error field gradients in quadrupole magnets

Random error sextupole field gradients in sextupole magnets

Random normal quadrupole field errors in dipole magnets

Random normal sextupole field errors in dipole magnets

Random normal sextupole field errors in quadrupole magnets

Random normal octupole field errors in quadrupole magnets

Tolerance Limit

$\begin{array}{ll}1.0 & \mathrm{mrad} \\ 0.1 & \mathrm{~mm} \\ 0.2 & \mathrm{~mm} \\ 2 \times 10^{-3} & \\ 4 \times 10^{-3} & \\ 2 \times 10^{-2} & \\ \mathrm{~b}_{1}=4 \times 10^{-4} & \mathrm{~cm}^{-1} \\ \mathrm{~b}_{2}=3 \times 10^{-4} & \mathrm{~cm}^{-2} \\ \mathrm{~b}_{2}=1 \times 10^{-3} & \mathrm{~cm}^{-2} \\ \mathrm{~b}_{3}=3 \times 10^{-4} & \mathrm{~cm}^{-3}\end{array}$

$0.1 \mathrm{~mm}$ produces orbit distorticns of $5 \mathrm{~mm}$ at the sextupoles, ${ }^{3}$ leading to a sizable detuning and consequent dynamic aperture reduction which is right at the tolerance limit. Within the tolerance, the closed orbit and beta and dispersion functions are acceptable [1]. Moreover, we showed that with groups of quadrupoles of alternating polarities aligned at high precision on girders, the alignment tolerance for the girders is greater than the tolerance for individual quadrupole misalignments by a factor of 5 . This result is also predicted analytically [4]. Finally, as shown in [1], the designed correction dipole system effectively restores the dynamic aperture for transverse quadruple misalignments as large as lmm rms.

\section{ACKNOWLEDGEMENTS}

We are indebted to Steve Kramer for many useful discussions and help with the codes used in our simulations.

3In the APS storage ring, this amplificalion factor, defined as the ratio of the rms quadrupole displacements and the rms orbit distortions, is of the order of $\mathbf{5 0}$.

\section{REFERENCES}

[1] H. Bizek, E. Crosbie, E. Lessner, L. Teng, J. Wirsbinski, "Effects of Construction and Alignment Errors on the Orbit Functions of the Advanced Photon Source Storage Ring," to appear in the 1991 Particle Accelerators Conference Record.

[2] A. Wrulich, "RACETRACK -.. A Computer Code for the Simulation of Non-Linear Particle Motion in Accelerators," DESY 84-026, 1984.

[3] S. Kramer, "Changes in the Program RACETRACK," unpublished report, 1990.

[4] E. Lessner and F. Mills, "Effects of Random Quadnupole and Girder Errors on the Closed Orbit in the APS Storage Ring," unpublished report, 1990. 